Research Article

\title{
Automatic Repair Method for D2D Communication Routing Buffer Overflow Vulnerability in Cellular Network
}

\author{
BaoPing Yang and Kun Jiang \\ Huanggang Normal University of Physics and Telecommunications, Huanggang Normal University, Huanggang 438000, \\ Hubei, China \\ Correspondence should be addressed to Kun Jiang; jiangkun@hgnu.edu.cn
}

Received 19 October 2021; Revised 5 November 2021; Accepted 17 November 2021; Published 20 December 2021

Academic Editor: Bai Yuan Ding

Copyright (C) 2021 BaoPing Yang and Kun Jiang. This is an open access article distributed under the Creative Commons Attribution License, which permits unrestricted use, distribution, and reproduction in any medium, provided the original work is properly cited.

\begin{abstract}
Repairing D2D communication routing buffer overflow in a cellular network is of great significance in improving communication quality and security. Due to the increase of user usage, the communication data are easy to exceed the boundary of the buffer, resulting in the reduction of covered data information. The traditional repair methods mainly repair through the characteristics of covered data information, ignoring the impact of network topology information transmission delay and packet loss during calculation, resulting in the problem of low communication security. A cellular network routing buffer overflow repair algorithm based on the homomorphic analysis of node residual energy is proposed; the cellular network D2D communication routing protocol is designed; the cellular network D2D communication protocol path index is determined; then, the cellular network D2D communication routing protocol is designed by analyzing node residual energy; and the cellular network D2D communication network routing optimization method based on AHP is designed. Big constructs the energy model of cellular network D2D communication network, solves and sets the routing optimization objective function, realizes the control of network routing, and repairs the buffer overflow. The experiment results show that the improved method can effectively reduce the packet loss rate of communication data, improve the anti-interference ability of the system, and ensure the security of network communication.
\end{abstract}

\section{Introduction}

Cellular network D2D communication usually takes electromagnetic wave as the carrier to receive and transmit network signals. A cellular network electromagnetic wave communication system plays an important role in implementing long-range precision strike and target precision guidance [1]. In the electromagnetic wave communication system of the cellular network, because the routing protocol evolves with the mobile location algorithm, the routing location cannot meet the needs of useful QoS, resulting in the buffer overflow problem, which needs to be repaired $[2,3]$. Therefore, it is of great significance to study the automatic repair method of $\mathrm{D} 2 \mathrm{D}$ communication routing buffer overflow vulnerability in the cellular network.

In literature [4], due to the lack of software execution information, static buffer overflow detection technology often reports too many false positives. Manually checking all static warnings is time-consuming. Bovinspector, a framework for automatically verifying static buffer overflow warnings, is proposed, and suggestions are provided for $\mathrm{C}$ programs to automatically repair real buffer overflow warnings. Given the program source code and static buffer overflow warning, Bovinspector first performs warning reachability analysis. The Bovinspector then symbolically executes the source code under the guidance of reachability warnings. Each reachable warning is verified and classified by checking whether all path conditions and buffer overflow constraints can be met at the same time. For each verified real warning, Bovinspector will provide suggestions and automatically repair it with 11 repair strategies. However, this method does not consider the characteristics of cellular network D2D communication, so the repair performance needs to be further improved. In [5], in case of earthquake, 
debris flow, and other disasters, the communication infrastructure may fail, resulting in blind areas and inconvenient communication for residents. Therefore, a new scheme to connect these infrastructure fault areas, namely, energyaware device-to-device communication scheme (need), is proposed. The proposed scheme uses cluster technology to connect users in the infrastructure failure area that often cannot directly access the cellular network. Compared with traditional clustering methods, it increases the process of determining candidate cluster heads before determining the final cluster heads. Based on the location and residual energy, the final CHS is selected from the candidate CHS, and the dual CHS in the cluster operates alternately to share the communication cost. In addition, in order to improve the routing efficiency, an improved ant colony algorithm (Maca) is proposed. The experiment results show the effectiveness of the proposed demand scheme in energy consumption and energy balance, and show that the scheme significantly prolongs the lifetime of the whole network. However, this method has the problem of large amount of calculation. Reference [6] studies multihop device-to-device (D2D) communication for cell coverage expansion. Considering the inband underlay D2D mode, the purpose is to meet the signal-to-noise ratio requirements of the pre-allocated resource block $(\mathrm{RB})$ on the downlink connection, the signalto-noise ratio requirements of the pre-allocated $\mathrm{RB}$ on each D2D side chain connection, and the maximum allowable interference at the base station receiver on all uplink RBS. While meeting these requirements, power control and routing are performed to minimize user equipment energy consumed in the system. An optimization problem is proposed, which is a mixed integer nonlinear programming and solved by Generalized Benders Decomposition (GBD). GBD decomposes the formula into main subproblem, auxiliary subproblem, and feasibility subproblem. However, this method is to repair vulnerabilities, and the repair performance needs to be further analyzed. In reference [7], network security is one of the main issues to be considered when designing a smart grid communication network (SGCN). However, due to the openness and unpredictability of wireless network, it is often vulnerable to different antagonistic attacks. It uses vulnerabilities to launch cross-layer attacks in the process of data transmission. In order to solve this problem, a new trust-based routing framework is proposed. The framework uses Bayesian reasoning to calculate direct trust and, combined with the evidence of reliable neighbors, uses the D-S theory to calculate indirect trust. Analytic hierarchy process (AHP) calculates the credibility and trust of nodes by using cross-layer indicators such as transmission rate, buffer capacity, and received signal strength. In addition, combining the fuzzy theory with BDS-AHP, fairness is considered when cross-layer metrics are used to calculate link trust to achieve reliable routing. Through a large number of experiments, the performance of the proposed fuzzy-based trusted routing algorithm (FBDSAHP) when malicious nodes initiate packets is evaluated. However, when calculating the link trust, this method is vulnerable to interference nodes, resulting in the decline of repair performance.
It can be seen from the analysis that the traditional method uses the electromagnetic wave diversity reception predictive control algorithm to repair the buffer overflow [8]. Because the diversity equalizer in QoS space shares the same channel with the diversity receiver, the edge space gain is very large. For QoS requirements, a cellular network routing buffer overflow recovery algorithm based on node residual energy homomorphism analysis is proposed to improve the algorithm. The performance test by experiment shows the superiority of the algorithm in this article.

\section{D2D Communication Routing Buffer Overflow in Cellular Network}

Establishing network topology, solving robust coefficient, and updating routing are the whole process of graph routing buffer overflow repair. In the cellular network D2D communication system, there are many routing hole space branches, resulting in poor repair effect of D2D communication routing buffer overflow. First, according to the cellular network D2D communication structure, the initial network topology is obtained, as shown in Figure 1.

In order to ensure the safe operation of cellular network D2D communication, each link index in the path must be optimized by calculating the path index of the cellular network D2D communication protocol [9]. The D2D communication protocol path index of the cellular network selected in this article includes the reliability index and the stability index. Among them, the reliability index can judge the quality of cellular network D2D communication, and the stability index can judge the data transmission stability of cellular network D2D communication.

The reliability index can be subdivided into link quality and path quality, and these two indexes can be expressed by calculation. If the expression of link quality reliability index is $L$, the following formula can be obtained:

$$
L=\frac{N}{X},
$$

where $N$ refers to the number of communication information correctly received by the link and $X$ is the total number of messages sent by the sender. Through formula (1), the link quality reliability is obtained to reflect the periodic propagation correct probability of cellular network D2D communication. On this basis, let the expression of path quality reliability index be $P$, and the following formula can be obtained:

$$
P=\prod L^{K}
$$

where $K$ refers to the first transmission success rate of cellular network D2D communication. The D2D communication reliability index of the cellular network can be calculated by formula (1) and formula (2). For the calculation of stability index, this article adopts the link failure estimation model, sets up the evaluation node in the cellular network D2D communication, and takes the node as the center to establish the spatial three-dimensional coordinate axis. The link failure time is determined by calculating the 


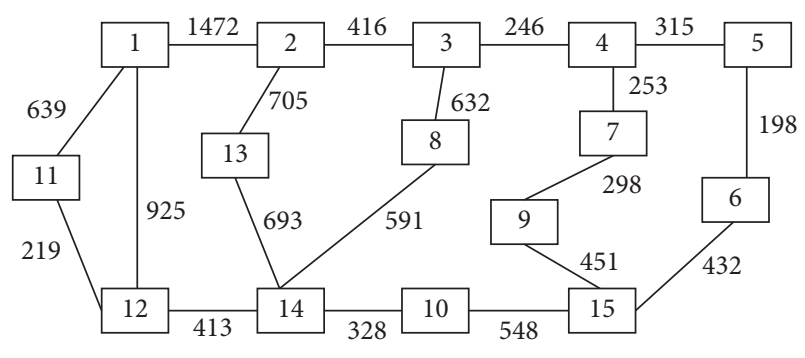

Figure 1: Communication network topology.

distance between the node and the communication transmission speed vector of cellular network D2D [10]. Using the cosine theorem, this process can be expressed by calculation. If the objective function is $T$, the following formula can be obtained:

$$
T=\frac{d \cos \theta+\sqrt{R^{2}-(d \sin \theta)^{2}}}{|v|},
$$

where $d$ refers to the communication range radius of cellular network D2D; $R$ refers to the coordinates of the evaluation node; $v$ refers to the transmission speed of cellular network $\mathrm{D} 2 \mathrm{D}$ communication; and $\theta$ is the angle of transmission motion. According to formula (3), when $\theta$ is greater than $90^{\circ}$, the distance between the node and the communication transmission speed vector of cellular network D2D is far, and the link failure time is short; when $\theta$ is less than $90^{\circ}$, the distance between the node and the communication transmission speed vector of cellular network D2D is close, and the link failure time is long. It can be seen that the greater the $T$ value, the higher the communication stability of cellular network D2D; On the contrary, the smaller the $T$ value, the lower the communication stability of cellular network D2D. The correlation index of cellular network D2D communication refers to the correlation degree before reliability and stability. Generally, it can be expressed by intersecting path and disjoint path. The intersecting path indicates that the cellular network D2D communication is greatly disturbed by the outside world and there is a risk of chain breaking, resulting in low reliability and stability of cellular network D2D communication [11]. Disjoint path means that the cellular network D2D communication is less disturbed by the outside world and there is no risk of chain breaking, resulting in high reliability and stability of cellular network D2D communication.

A program is usually composed of multiple subroutines (modules). The larger the program size, the more the modules. After compiling a program, it is divided into three areas in the memory to store the program code area, data area, and stack area. Among them, various variables and buffer areas defined in the program are stored in the data area, and the return address of the calling subroutine is stored in the stack area. When the control returns from the subroutine, return to the main control program according to the address indicated at the top of the stack.

When writing a program in the C language, the input function is generally used to obtain the name entered by the user. When the user tries to input more name characters that can be processed by the buffer into the buffer, if there is no buffer cross-border check mechanism in the program, the buffer overflow will occur when an ultralong string is input. For example, if char name is defined in the program written by the programmer, when a string longer than 15 bytes is input, the character buffer will overflow. By judging the reliability index and stability index, it is judged that there is a vulnerability in the cellular network D2D communication, and then, the repair is completed according to the node residual energy analysis.

\section{Homomorphism Analysis of Node Residual Energy}

3.1. User Transmit Power Adjustment. In the D2D communication system, the communication link between adjacent users is allowed to be established directly, so as to complete the transmission of information. In the process of establishing a direct link, although it does not pass through the relay forwarding of the base station [12], the base station needs to control the whole process through the center or auxiliary control. In terms of network control, the D2D communication network architecture can be divided into network center control network architecture and network auxiliary control network architecture. The D2D network communication architecture is given in Figure 2.

3.1.1. Network Center Control Network Architecture. In the network center control network architecture, the whole process of D2D communication is controlled by the network management center (base station). According to the user's environment, the base station can quickly complete the user discovery process and judge whether adjacent users establish a direct communication link. Before determining to establish a direct communication link, the base station also needs to allocate communication resources to D2D users and configure network parameters. In addition, under the centralized control mode, the base station can efficiently manage the interference between users [13, 14], which is conducive to improving the network performance. However, this central control mode requires a lot of signaling overhead, which is difficult to obtain in the actual wireless network.

3.1.2. Network Auxiliary Control Network Architecture. In the network-aided control network architecture, firstly, for D2D users, it needs to be able to perceive the relevant information of other users and the use of network resources according to its own business needs, so as to independently complete device discovery and dynamically adjust its transmission power. In addition, the base station can master the information in the whole network [13], assist D2D users in resource allocation, identity authentication and other functions, and then establish a direct communication link between users. In this way, D2D autonomy has distributed characteristics, and its communication process can be completed with a small amount of signaling overhead. 


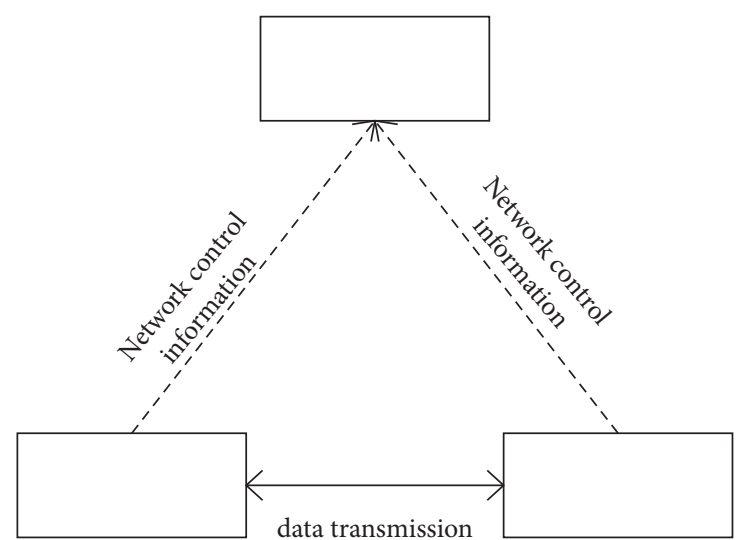

(a)

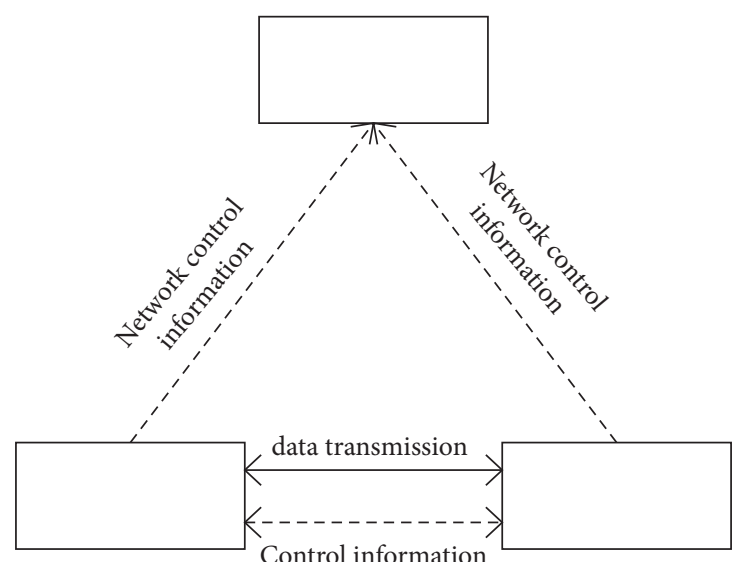

(b)

Figure 2: D2D communication network architecture. (a) Network center control network architecture. (b) Network auxiliary control network architecture.

Moreover, through the auxiliary role of the base station, the uncontrollability caused by the complete autonomy of D2D communication is avoided. However, compared with the network center control network architecture, this network architecture is not efficient enough.

There are three working modes of D2D communication network:

(1) Cellular Mode. The cellular mode here is the same as the traditional cellular mode. Because users do not have the conditions to establish a direct communication link, users can only complete data transmission through the base station. Compared with the D2D communication mode [15], the cellular mode avoids interference between users, but the spectrum resource utilization is low. The specific structure is shown in Figure 3.

(2) Private Mode. For the dedicated mode, two adjacent users who are qualified to establish D2D communication links can directly establish communication links to complete the transmission of information. However, the reserved spectrum resources need to be allocated to D2D users, and these reserved frequency bands, including uplink and downlink frequency bands, are orthogonal to each other, which can avoid mutual interference between users. Moreover, compared with the cellular mode [16], the dedicated mode only needs one downlink spectrum or one uplink spectrum to complete D2D communication, hence high resource utilization.

(3) Sharing Mode. In the sharing mode, D2D users multiplex the authorized frequency band in the cellular network for data transmission. Moreover, D2D users can multiplex the spectrum resources of downlink users and uplink users. Compared with cellular mode and dedicated mode, shared mode has the advantage of high-frequency spectral efficiency. However, D2D multiplexing the spectrum resources of cellular users will cause interference between two types of

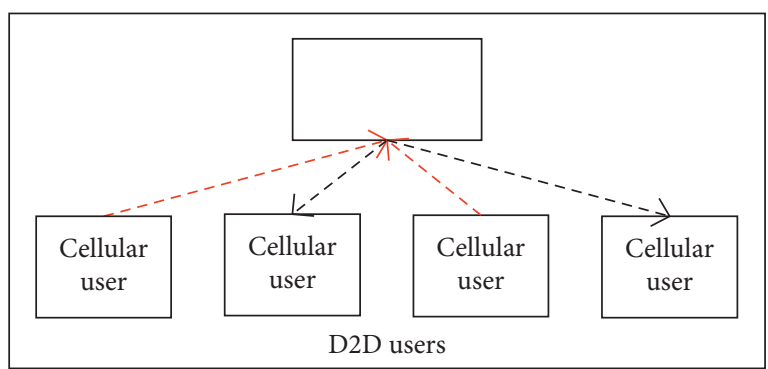

Figure 3: Cellular mode.

users. Effective resource allocation algorithm and interference management scheme are needed to avoid or reduce these interferences $[17,18]$.

In a complete $\mathrm{D} 2 \mathrm{D}$ communication process, it mainly includes three steps: (1) device discovery, (2) communication establishment, and (3) data transmission. A cellular user will find the users communicating with it autonomously or through the base station according to their own business needs and the relevant information of other users, then complete the establishment of communication link under the control of the base station, and finally realize the data transmission of two adjacent users. The specific implementation process is shown in Figure 4. Taking two adjacent users as an example, the whole implementation process of D2D communication is analyzed and studied.

(1) User A prepares to communicate with user B according to his own needs. At this time, user A sends a request to establish a communication link to the base station in his cell, which includes some information of user B [19].

(2) After receiving the request from user $A$, the base station starts to collect the relevant information of user $B$ and find the location of user B, and then, the base station sends D2D communication measurement signaling to two users [20]. 


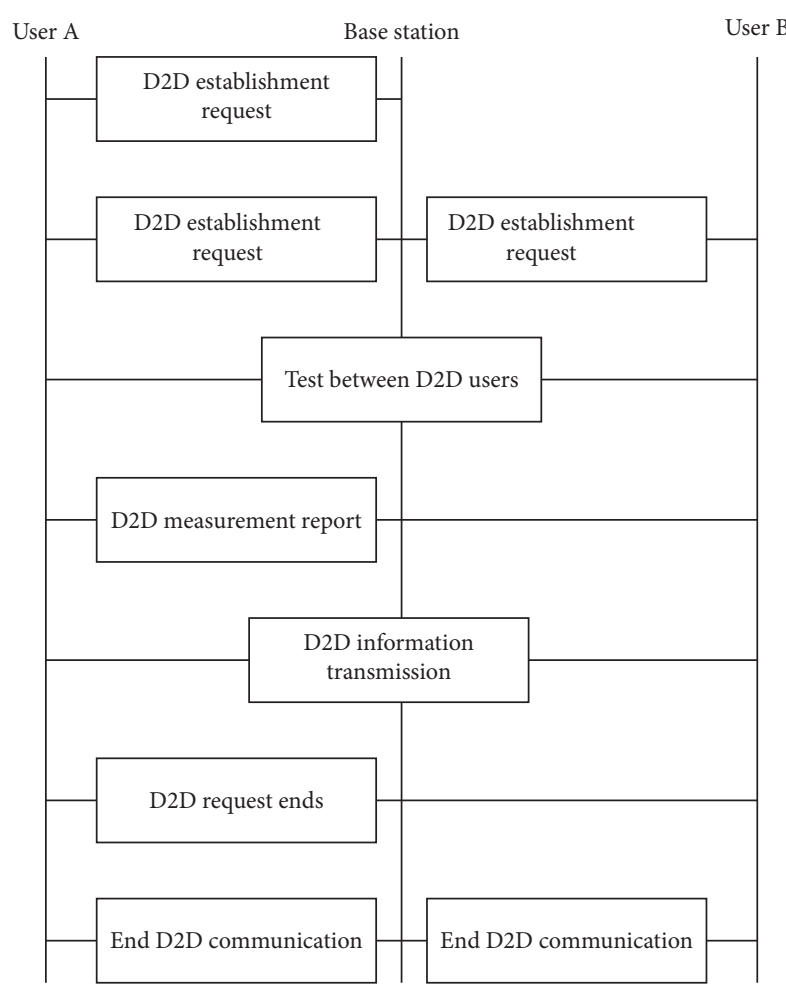

FIGURE 4: D2D communication implementation flowchart.

(3) After receiving the measurement signaling, user A and user B start to measure the channel state information between their two users, which is used by the base station to judge whether the conditions for establishing D2D communication link can be met between the two users, send the test results to the base station through user A or user B, and record the test results.

(4) After receiving the channel state information between the two users, the base station judges whether user A and user B can establish a D2D communication link according to the predetermined conditions for establishing a D2D communication link. If the conditions are true, the base station sends the judgment result to the two users.

(5) After receiving the notification, user A and user B establish a direct communication link to complete data transmission.

(6) After user A and user B complete data transmission, the user sends a request to the base station to end D2D communication.

(7) After receiving the request to end D2D communication, the base station will change the network parameter configuration, withdraw the determination information of D2D communication between user $\mathrm{A}$ and user $\mathrm{B}$, and end $\mathrm{D} 2 \mathrm{D}$ communication between user $\mathrm{A}$ and user $\mathrm{B}$.

In the D2D communication system, compared with unicast communication mode, multicast communication mode is that multiple D2D users are combined together, and they share the same content and can transfer information to multiple D2D users at the same time. In addition, due to the introduction of multicast technology, D2D users can form multicast communication with multiple users and transmit the same data resources to other users at the same time, which can improve the utilization efficiency of network resources and reduce the time of establishing communication between D2D users. As shown in Figure 5, multiple D2D users form a multicast communication group. In each multicast communication group, one user can transmit data to one of them or send data to multiple D2D users at the same time.

(4) Broadcast Communication Mode. D2D broadcast communication means that when a D2D user transmits information, other users away from the user can receive the information.

With the development of communication technology and the improvement of people's living standards, the number of users in the communication network is increasing rapidly, and the load of the base station is increasing. As a result, the base station cannot guarantee to provide good service quality for users within the coverage. As one of the key technologies of LTE and future 5G, D2D communication allows adjacent devices to communicate directly under the control of the cellular system. The data between users does not go through the base station routing, which can effectively reduce the base station load [21] and shorten the end-to-end delay. In addition, the D2D communication under the cellular network allows D2D users to reuse channel resources of cellular users, thereby significantly improving high-frequency spectrum utilization and system capacity. D2D communication technology not only brings many benefits, but also brings new problems, that is, $\mathrm{CO}$ frequency interference caused by spectrum sharing. Therefore, effective interference management and resource allocation schemes must be developed to solve the above interference problems [22] and maximize the throughput of D2D links in the system on the premise of resource constraints.

3.2. Design of Electromagnetic Wave Multiple Routing Protocol in Cellular Network. The adopted channel model not only considers the path loss, but also considers the fast fading caused by multipath effect and the slow fading caused by shadow effect. Therefore, the channel gain between the cellular user and the D2D user receiver can be expressed as

$$
h_{c d}=k \cdot \delta_{\text {cld }} \cdot \xi_{\text {cld }} \cdot d_{\text {cld }}^{-\alpha},
$$

where $k$ represents the path loss coefficient; $\alpha$ represents path loss index; $\delta_{\text {cld }}$ represents the fast fading factor obeying exponential distribution; $\xi_{\text {cld }}$ represents the slow fading factor obeying log positive attitude distribution; and $d_{\text {cld }}^{-\alpha}$ represents the distance between the cellular user and the D2D user receiving end.

It is assumed that there are $D$ pairs of D2D users in the system, and $C$ cellular users use mutually orthogonal channels for communication. When D2D user $d$ multiplexes 


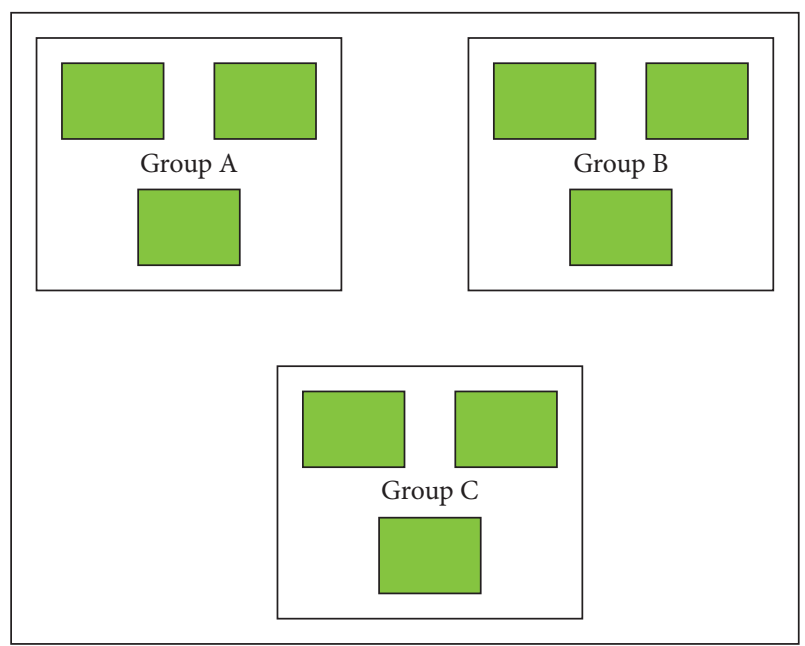

Figure 5: D2D multicast communication mode.

the channel resources of cellular user $i$, the signal-to-interference noise ratio and rate of cellular user $i$ are, respectively,

$$
\begin{aligned}
& \gamma_{i}=\frac{P^{i} h_{c b}}{P_{d}^{i} h_{d c}+N_{0}}, \\
& r^{i}=\log _{2}\left(1+\gamma_{i}\right) .
\end{aligned}
$$

The SINR and rate of D2D user $d$ are

$$
\begin{aligned}
& \gamma_{d}^{i}=\frac{P_{d}^{i} h_{d d}}{P^{i} \log _{2}\left(1+\gamma_{d}^{i}\right)}, \\
& r_{d}^{i}=\log _{2}\left(1+\gamma_{d}^{i}\right) .
\end{aligned}
$$

When D2D users and cellular users share the same channel, cellular users and D2D users will produce common channel interference, and the closer the distance, the greater the interference. Generally, the number of users performing $\mathrm{D} 2 \mathrm{D}$ communication at the same time in the cell is less than the number of cellular users. Therefore, there may be multiple cellular channel resources as potential multiplexing objects for D2D users. In order to ensure the QoS requirements of D2D users, the base station allows D2D users to reuse the channel resources only when the interference of cellular users to D2D users is less than a certain threshold. This section defines the areas where cellular users are restricted from multiplexing:

$$
P_{c, \max } h_{c d} \leq I_{c, d}
$$

Given the channel resource set multiplexed by D2D users, the transmission power of D2D users is optimized through power control to maximize the throughput of D2D link under resource constraints [23]. From the previous step, the reusable potential cellular channel of any D2D user $D$ can be determined, and it can be obtained that the set of

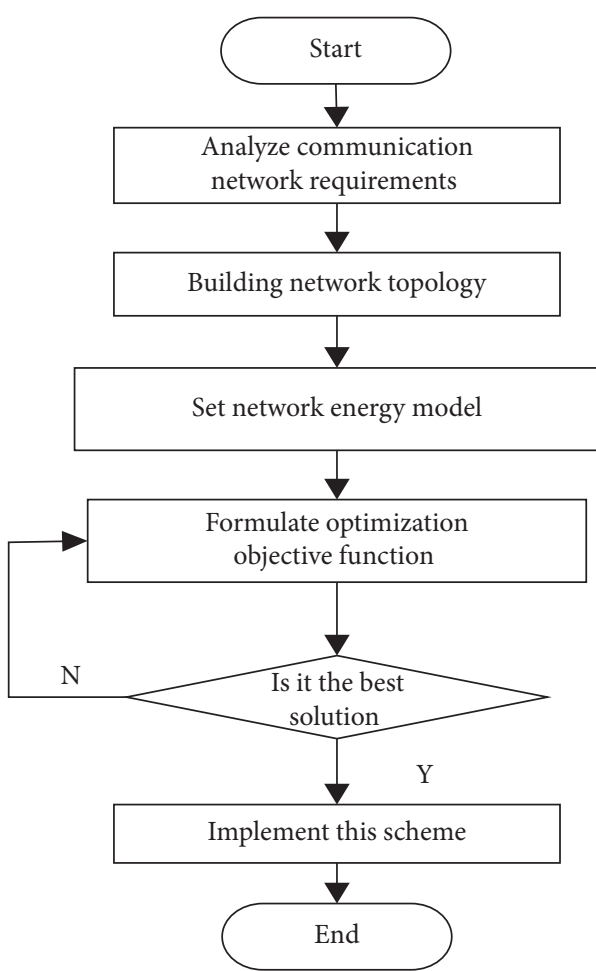

FIGURE 6: Route optimization process of the cellular network D2D communication network.

resources $D_{d}$ can reuse is $S_{d}$. Therefore, the optimization objective can be expressed as

$$
\left\{\begin{array}{l}
\max u_{d}^{k}=\sum_{i \in S_{d, k}} \log _{2}\left(1+\frac{P_{d}^{i} h_{d d}}{P^{i} \log _{2}\left(1+\gamma_{d}^{i}\right)}\right) \quad 0 \leq P_{d}^{i} \leq P_{c, \max }, \\
\sum_{i \in s_{d, k}} \leq P_{c, \max } .
\end{array}\right.
$$

According to the optimization objectives and constraints, the water injection algorithm is used to allocate power to D2D users. According to the Karush-Kuhn-Tucker (KKT) condition,

$$
\begin{aligned}
L\left(P_{d}^{i}, \alpha, \beta\right)= & \sum_{i \in S_{d, k}} \log _{2}\left(1+\frac{P_{d}^{i} h_{d d}}{P^{i} \log _{2}\left(1+\gamma_{d}^{i}\right)}\right) \\
& -\alpha\left(P_{d}^{i}-P_{c, \max }\right)-\beta\left(\sum_{i \in S_{d, k}} P_{d}^{i}-P_{c, \max }\right) .
\end{aligned}
$$

To calculate the partial derivative, there are

$$
P_{d}^{i}=\left[\delta-\frac{1}{H}\right]^{+}
$$

of which 


$$
\begin{aligned}
{[\varphi]^{+} } & =\max \{0, \varphi\}, \\
H & =\frac{h_{d d}}{P^{i} \log _{2}\left(1+\gamma_{d}^{i}\right)} .
\end{aligned}
$$

Through the power control in the previous step, we can know the maximum throughput and corresponding transmission power of any resource set multiplexed by D2D users at a set time. At the same time, in the process of channel allocation, the power adjustment of users on different channels will be completed through the scheme of power control part.

\subsection{Design of Routing Optimization Method of Cellular} Network D2D Communication Network Based on AHP. The traditional daily cellular network D2D communication network routing often has the problem of excessive energy consumption in use, which affects the stability of the cellular network D2D communication network. Therefore, set the cellular network D2D communication network routing optimization method based on AHP to deal with it. The specific routing optimization process is shown in Figure 6.

The routing in the communication network is processed in detail according to the steps set in 6 . In order to ensure the reliability of the optimization results, the calculation accuracy is set in the calculation process.

\subsubsection{Building a Cellular D2D Communication Network} Energy Model. In order to control the energy consumption of the communication network, a cellular network D2D communication network energy model is constructed. Before building the energy model, complete the setting of the network model. The nodes in this network model have the following characteristics: the node number is single and random [24, 25]. All nodes have corresponding fixed positions, and node energy is limited. The location of the base station is fixed and is not considered in the energy model. All nodes in the network are isomorphic nodes with the same initial energy and communication radius. All nodes have the ability to directly realize data transmission with the base station. Nodes have certain data integration ability, and their current residual energy can be obtained through calculation $[26,27]$. The data transmission capacity and power of all nodes can be adjusted, and the data fusion technology can be used to reduce the amount of data transmission. The nodes can determine the transmission distance according to the strength of signal reception.

The first-order radio model is used as the basis of model design, and it is set as the form of free space model according to the characteristics of the cellular network D2D communication network [28]. Its network architecture has the following characteristics: when the distance between data transmitting node $r_{1}$ and data receiving node $r_{2}$ is less than $d_{1}$, the energy consumption of data transmission power amplification is directly proportional to $d_{1}^{2}$. Set the transmitting node to send the data of $o$ bits to the receiving node with distance $d_{2}$. The energy consumption consists of transmission power consumption and power amplification consumption, which can be expressed as follows:

$$
W_{i}(h, d)=\left\{\begin{array}{l}
h W_{\text {elec }}+h W_{f} d^{2}, \\
h W_{\text {elec }}+h W_{\text {amp }} d^{4},
\end{array}\right.
$$

where $W_{\text {elec }}$ represents the energy consumption of the transmitting circuit or receiving circuit when transmitting or receiving 1 bit data information and the free space signal amplification factor is $W_{f}[29,30]$. When there are too many channels in the communication network, the signal amplification factor is $W_{\text {amp }}, d$ represents the distance between the transmitting node and the receiving node, and the data length in the communication network is set to $h$ bit, and then $d_{1}$ is

$$
d_{1}=\sqrt{\frac{\alpha_{f}}{\alpha_{m p}}},
$$

where $\alpha$ represents the straight-line distance of the node. The energy consumption formula of the node receiving $h$ bit data can be obtained from the above formula:

$$
W_{i}(h)=W_{\text {re-elec }}(h)=h W_{\text {elec }} \text {. }
$$

The node in the communication network transmits the collected data information to the cluster head node, performs data fusion through this node, and finally sends the fused data to the base station [31]. The process of data information fusion also needs to consume a lot of energy, so the energy consumption model of the $\mathrm{D} 2 \mathrm{D}$ communication network is established as the following formula :

$$
W_{D A}(h)=h \sum_{i} W_{i}(h),
$$

where $W_{D A}$ represents the energy consumption of fused $h$ bits data and $D A$ represents the data fusion coefficient. So far, the construction of the energy consumption model of cellular network D2D communication network is completed, and the optimization of network routing is realized on the basis of this model.

\subsubsection{Setting Routing Optimization Objective Function.} Since the structure of the communication network itself is difficult to change in practical application, in order to reduce the impact of link interruption on network operation, corresponding optimization strategies are proposed [32, 33]. Set the objective function in the optimization process as

$$
\min (\beta \bar{T}+\chi G J),
$$

where $\bar{T}$ represents the average transmission time of data information, $T_{i}$ represents the transmission delay of data packet $i, H$ represents the number of data packets, $G J$ is set as the service balance of the whole network, and the average data transmission delay of the communication network is

$$
\bar{T}=\sum_{i=1}^{H} \frac{T}{H_{i}},
$$


where $H_{i}$ represents the number of services on the $i$-th link, $m$ represents the number of links in the communication network, $T_{\max }$ represents the longest delay in data transmission in the communication network, $H_{\max }$ represents the maximum number of services carried by the communication network link, and $\beta$ and $\chi$ are constant terms in the calculation process. The service balance degree of the communication network can be obtained by the following formula :

$$
G J=\sqrt{\sum_{i=1}^{m} \frac{\left(H_{i}-\sum_{i=1}^{H} H_{i}\right)^{2}}{m}} .
$$

The energy consumption and data transmission delay are set as the routing optimization objectives. It is applied to the model set above to remove the interrupted link $x$ and obtain a new network topology. The shortest path algorithm is used to find the shortest transmission path for packet $A[34,35]$, then the optional path set of packet can be expressed as $P\left(p_{i}, \quad i=1,2, \ldots, n\right)$, and the fuzzy analytic hierarchy process is used to obtain the optimal communication network routing combination $P_{s}$. On the basis of the above calculation, the adjacent nodes with the shortest route are introduced to generate the subshortest route path and finally form the shortest route set as an alternative to route optimization. In the process of communication, when the signals are separated, the high-order Bessel function detection and control technology are introduced to make the network branch signals add in phase during communication, offset the phase offset of signal fading in the process of network communication, and finally achieve the purpose of routing buffer overflow repair.

\section{Experiment and Performance Test}

In order to verify the effect of this algorithm in repairing routing buffer overflow in the cellular network, experiment verification is carried out. The TOSSIM simulator of the TinyOS system is used to construct the cellular network experiment model, and the delay, energy efficiency, and the number of contracts are used to test the superiority of the method proposed in this article. In the experiment parameter setting, the routing Grace period is $2 \mathrm{~ms}$, and the mac-pr deployment is adjusted. The network routing frequency is $7.8 \mathrm{~Hz}$, and only $5.5 \mathrm{~s}$ is relayed each time. The mac-pr can accommodate 400 nodes. The network routes under different bandwidths, CLS lengths, and power losses are selected. See Table 1 for specific parameters.

4.1. Node Residual Energy. The test results of node residual energy of the automatic repair method for D2D communication routing buffer overflow vulnerability in the cellular network designed in literature [4], literature [7], and this article are shown in Figure 7.

According to the two test results shown in Figure 7, it can be seen that in the method of reference [4], due to the failure of the inner ring node, the distance of the outer ring node sending information is too large, and its energy is
TABLE 1: Network routing design parameters.

\begin{tabular}{lccc}
\hline \multicolumn{2}{c}{$\begin{array}{c}\text { Paragraph A (ECA test } \\
\text { indicators) }\end{array}$} & $\begin{array}{c}\text { Paragraph B (ECA test } \\
\text { indicators) }\end{array}$ \\
\hline Parameter & Index & Parameter & Index \\
Frequency & $7.8 \mathrm{~Hz}$ & Frequency & $7.8 \mathrm{~Hz}$ \\
Bandwidth & $130 \mathrm{Mhz}$ & Bandwidth & $80 \mathrm{Mhz}$ \\
CLS length & $1.9 \mathrm{~ms}$ & CLS length & $1.1 \mathrm{~ms}$ \\
Breakpoint loss & $11 \%$ & Breakpoint loss & $13 \%$ \\
WSN receiving & LC & WSN receiving & LA \\
antenna & $93 \%$ & antenna & Receiving rate \\
Receiving rate & \multicolumn{3}{c}{$96 \%$} \\
\hline
\end{tabular}

exhausted first. The method in reference [7] does not cluster in the aggregation area, so the overhead is controlled and the protocol energy consumption is improved. This protocol uses the ant algorithm to search the communication route and find the global optimal transmission path.

\subsection{Routing Buffer Overflow Repair Results under Improved} Algorithm. Based on the above experiment environment and parameter design, the QoS oriented experiment of magnetic wave communication and routing buffer overflow repair in cellular network is carried out. The algorithm in this article is used to repair the overflow of D2D communication routing buffer in a slow cellular network. The repair results are shown in Figure 8.

By analyzing Figure 8 , it can be seen that the amplitude corresponding gain is the standard to measure the result of routing buffer overflow repair. After using this algorithm to repair the routing buffer overflow in the cellular network, the amplitude corresponding gain is improved compared with that before repair, which shows that the repair result is ideal, and proves the effectiveness of the routing buffer overflow repair algorithm proposed in this article.

Under the condition of the same number of iterations, the network communication packet loss rate before and after repair is used as the test index to obtain the comparison results of the network communication packet loss rate before and after communication network routing buffer overflow repair, as shown in Table 2.

Summarize the data in Table 2 and draw a broken line diagram of the network communication packet loss rate before and after communication network routing buffer overflow repair, as shown in Figure 9.

Analyzing Table 2 and Figure 9, with the increase in the number of iterations, the packet loss rate of cellular network D2D communication before and after routing buffer overflow repair also gradually decreases. However, under the same number of iterations, the packet loss rate of network communication after routing buffer overflow repair using this algorithm is always lower than that without repair. The average packet loss rate after routing buffer overflow repair using this algorithm is $19 \%$, whereas the average packet loss rate without routing buffer overflow repair is $30 \%$. Overall, it shows that the optimization of D2D communication routing buffer overflow repair in the cellular network can effectively reduce the packet loss 


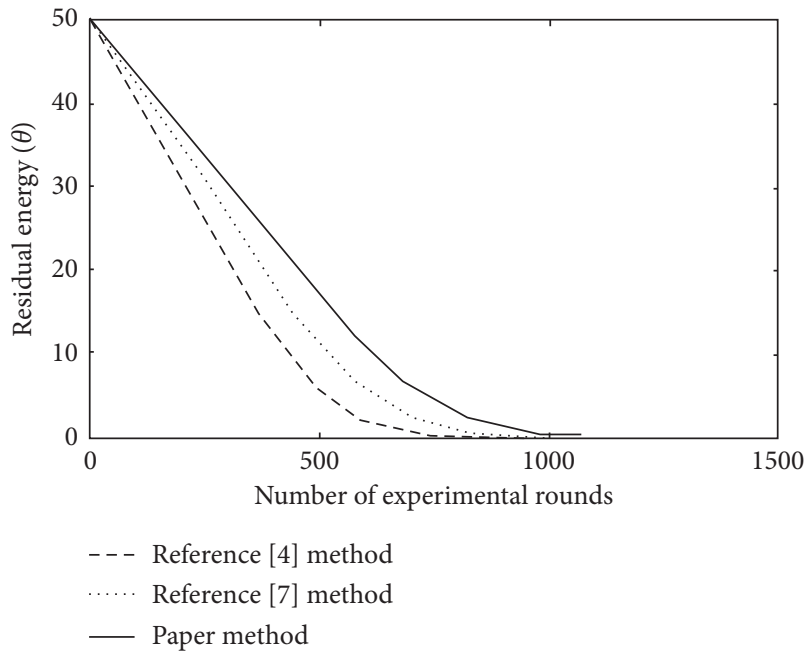

(a)

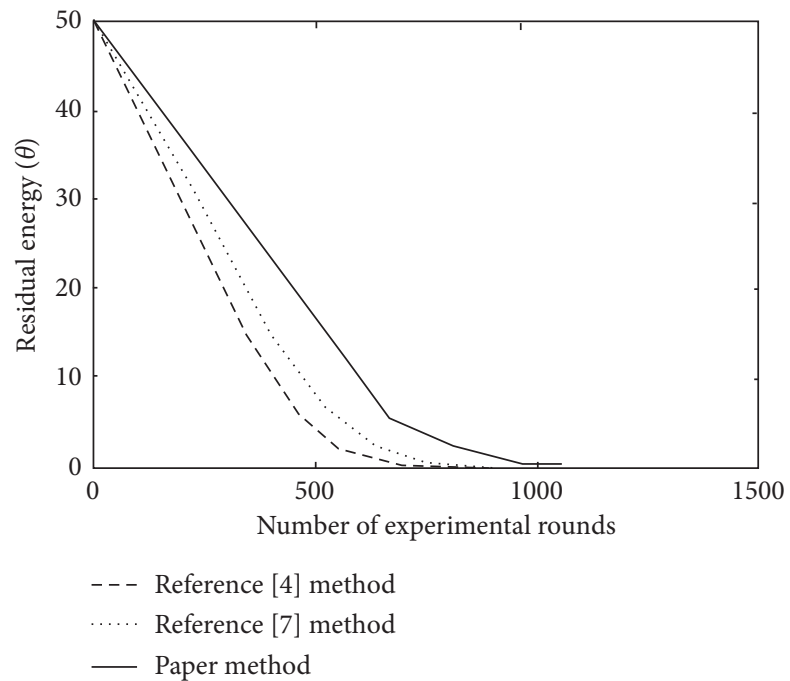

(b)

Figure 7: Comparison of $\theta$ value of node residual energy. (a) First test. (b) Second test.

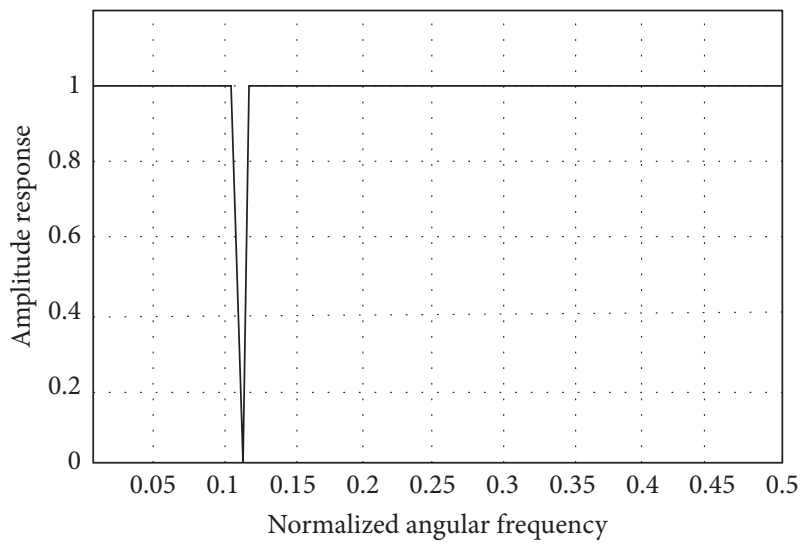

FIGURE 8: Routing buffer overflow repair results.

TABle 2: Comparison results of the network communication packet rate before and after repair.

\begin{tabular}{lcc}
\hline Number of iterations & Using buffer repair (\%) & Buffer repair not used (\%) \\
\hline 0 & 52 & 85 \\
10 & 43 & 55 \\
20 & 33 & 42 \\
30 & 20 & 37 \\
40 & 18 & 32 \\
50 & 15 & 20 \\
60 & 12 & 18 \\
70 & 5 & 15 \\
80 & 3 & 12 \\
90 & 2 & 10 \\
100 & 1 & 3 \\
\hline
\end{tabular}

rate of communication data, improve the anti-interference ability of the system, ensure the network communication security, and meet the QoS standards and requirements.
4.3. Communication Performance Comparison under Overflow Repair. Under different experimental times, the communication performance of cellular network D2D before 


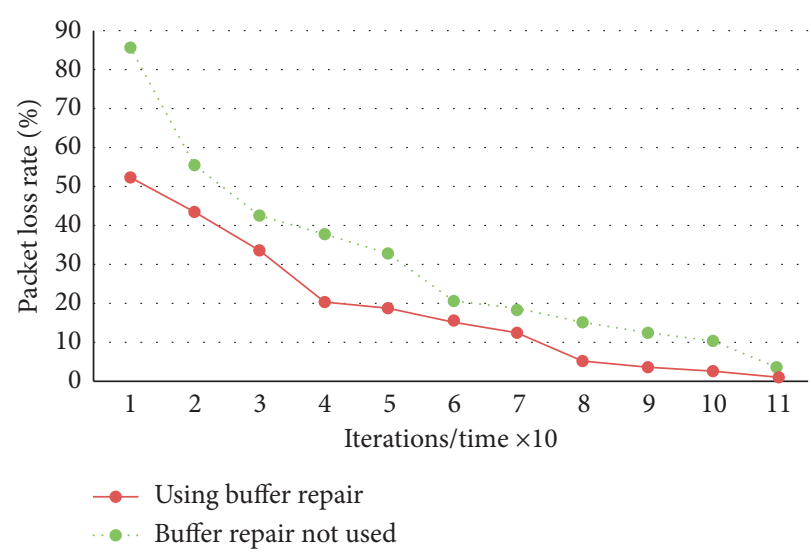

FIGURE 9: Broken line diagram of network communication packet loss rate comparison.

TABLE 3: Communication performance after the repair of routing buffer overflow in the communication network.

\begin{tabular}{lccc}
\hline Number of experiments (times) & Blocking rate (\%) & Equilibrium (\%) & Stability (\%) \\
\hline 10 & 0.5 & 97 & 98 \\
20 & 0.8 & 96 & 97 \\
30 & 0.6 & 95 & 95 \\
40 & 0.4 & 97 & 96 \\
50 & 0.7 & 98 & 97 \\
60 & 0.6 & 96 & 98 \\
70 & 0.5 & 98 & 96 \\
80 & 0.3 & 96 & 96 \\
\hline
\end{tabular}

TABLE 4: Communication performance of the communication network before routing buffer overflow repair.

\begin{tabular}{lccc}
\hline Number of experiments (times) & Blocking rate (\%) & Equilibrium (\%) & Stability (\%) \\
\hline 10 & 4 & 69 & 71 \\
20 & 3 & 68 & 72 \\
30 & 5 & 65 & 74 \\
40 & 2 & 63 & 73 \\
50 & 6 & 67 & 71 \\
60 & 4 & 69 & 72 \\
70 & 5 & 68 & 74 \\
80 & 3 & 66 & 75 \\
\hline
\end{tabular}

and after routing buffer overflow repair is compared, and the communication blocking rate, communication route data distribution balance of cellular network D2D, and communication route data stability of cellular network D2D are compared. The results are shown in Tables 3 and 4 .

By analyzing Tables 3 and 4, it can be seen that in terms of communication blocking rate, the communication blocking rate before routing buffer overflow repair is large, and the average communication blocking rate is $4 \%$, while the blocking rate after routing buffer overflow repair is significantly reduced, and the average communication blocking rate is only $0.55 \%$, which is $86.5 \%$ lower than that before repair; In terms of routing data allocation balance, the routing data allocation balance before routing buffer overflow repair is small, and the average routing data allocation balance is $66.9 \%$, while the routing data allocation balance after routing buffer overflow repair is significantly increased, and the average routing data allocation balance is $96.9 \%$, which is $44.8 \%$ higher than that before repair. In terms of routing data allocation stability, the routing data allocation stability before routing buffer overflow repair is small, and the average routing data allocation stability is $72.6 \%$, which is poor. After routing buffer overflow repair, the routing data allocation stability has been significantly improved, and the average routing data allocation stability is $96.1 \%$, which is $32.3 \%$ higher than that before repair. It shows that the stability of routing data distribution after repair is good, because the cellular network routing buffer overflow repair algorithm based on node residual energy homomorphism analysis is proposed, the cellular network routing buffer overflow repair algorithm based on homomorphic analysis of node residual energy is proposed, the cellular network $\mathrm{D} 2 \mathrm{D}$ communication routing protocol is designed, the cellular network D2D communication protocol path index is determined, and then the cellular network D2D communication protocol is designed by analyzing node residual 
energy, and the cellular network D2D communication network energy model is constructed. Solve and set the routing optimization objective function to control the network routing and repair the buffer overflow.

\section{Conclusion}

In the cellular electromagnetic wave communication system, because the routing protocol evolves with the mobile location algorithm, the location routing cannot effectively meet the QoS requirements, resulting in the buffer overflow problem, which needs to be repaired. The traditional method uses the predictive control algorithm of electromagnetic wave diversity reception to repair the buffer overflow. Because the diversity equalizer in QoS space shares the same channel with the diversity receiver, the edge space gain is very large. For QoS requirements, a cellular network routing buffer overflow repair algorithm based on node residual energy homomorphism analysis is proposed to determine the path index of cellular network D2D communication protocol, then design the cellular network D2D communication routing protocol, build the cellular network D2D communication network energy model, solve and set the routing optimization objective function, and realize the control of network routing, Fix buffer overflow. The experiment results show that the proposed method can effectively reduce the packet loss rate of communication data, improve the communication performance and anti-interference ability of cellular network D2D communication, and ensure the communication security of the cellular network, and has a good application value.

\section{Data Availability}

The raw data supporting the conclusions of this article will be made available by the authors, without undue reservation.

\section{Conflicts of Interest}

The authors declare that they have no conflicts of interest regarding this work.

\section{Acknowledgments}

This work was supported by National College Students Innovation and Entrepreneurship Program Training Project: Design of Fire Prevention and Theft Device in College Dormitory (Project no. 201810514008), and National College Students Innovation and Entrepreneurship Program Training Project: Design and Implementation of Smart Home System Based on ARM (Project no. 202110514046).

\section{References}

[1] N. Anjum, Z. Yang, H. Saki, M. Kiran, and M. Shikh-Bahaei, "Device-to-device (d2d) communication as a bootstrapping system in a wireless cellular network," IEEE Access, vol. 7, no. 99, pp. 6661-6678, 2019.
[2] G. L. Shen, "Vulnerability of vulnerability defense control simulation of network system resource data," Computer Simulation, vol. 37, no. 4, pp. 308-311, 2020.

[3] F. T. Zuhra, K. Bakar, A. Ahmed, K. M. Almustafa, and N. Islam, "Lltp-qos: low latency traffic prioritization and qosaware routing in wireless body sensor networks," IEEE Access, vol. 7, no. 99, pp. 152777-152787, 2019.

[4] F.-J. Gao, Y. Wang, L.-Z. Wang, Z. Yang, and X.-D. Li, "Automatic buffer overflow warning validation," Journal of Computer Science and Technology, vol. 35, no. 6, pp. 14061427, 2020.

[5] H. Rong, Z. Wang, H. Jiang, Z. Xiao, and F. Zeng, "Energyaware clustering and routing in infrastructure failure areas with $\mathrm{d} 2 \mathrm{~d}$ communication," IEEE Internet of Things Journal, vol. 6, no. 5, pp. 8645-8657, 2019.

[6] A. Ibrahim, T. Ngatched, and O. A. Dobre, "Using bender's decomposition for optimal power control and routing in multihop d2d cellular systems," IEEE Transactions on Wireless Communications, vol. 18, no. 99, p. 1, 2019.

[7] D. Velusamy, G. Pugalendhi, and K. Ramasamy, "A crosslayer trust evaluation protocol for secured routing in communication network of smart grid," IEEE Journal on Selected Areas in Communications, vol. 38, no. 1, pp. 193-204, 2020.

[8] G. V. Abgaryan, "On the resonant passage of electromagnetic wave through waveguide with diaphragms," Lobachevskii Journal of Mathematics, vol. 41, no. 7, pp. 1315-1319, 2020.

[9] I. Singh and N. P. Singh, "Coverage probability analysis of device-to-device communication underlaid cellular networks in uplink over $\$ \$ \backslash$ kappa $-\mid \mathrm{mu} \$ \$ \kappa-\mu / \$ \$$ leta $-\backslash \mathrm{mu} \$ \$ \eta-\mu$ fading channels," International Journal of Wireless Information Networks, vol. 26, no. 1, pp. 39-47, 2019.

[10] O. A. Amodu, M. Othman, N. K. Noordin, and I. Ahmad, "Transmission capacity analysis of relay-assisted d2d cellular networks with interference cancellation," Ad Hoc Networks, vol. 117, no. 11, Article ID 102400, 2021.

[11] M. H. Khoshafa, T. Ngatched, M. H. Ahmed, and A. Ibrahim, "Improving physical layer security of cellular networks using full-duplex jamming relay-aided d2d communications," IEEE Access, vol. 8, no. 99, p. 1, 2020.

[12] A. N. Rao, B. R. Naik, and L. N. Devi, "On the relay node placement in wsns for lifetime maximization through metaheuristics," Materials Today: SAVE Proceedings, vol. 4, 2020.

[13] K. D. Rahi and Y. M. Hasan, "Utilizing device to device communication in cellular networks with the distance-based model selection method," IOP Conference Series: Materials Science and Engineering, vol. 870, Article ID 012116, 2020.

[14] A. Alnoman and A. S. Anpalagan, "Computing-aware base station sleeping mechanism in h-cran-cloud-edge networks," IEEE Transactions on Cloud Computing, vol. 9, no. 3, pp. 958-967, 2019.

[15] J.-A. He, L. Jia, L. Xu, and W. Chen, "Throughput maximization for multiple $\mathrm{d} 2 \mathrm{~d}$ group communications underlaying cellular networks," Wireless Communications and Mobile Computing, vol. 2020, Article ID 8833579, 10 pages, 2020.

[16] H. H. Su, W. B. Qu, and Y. Peng, "Uplink and downlink throughput optimization scheme for millimeter wave $\mathrm{d} 2 \mathrm{~d}$ communication," Procedia Computer Science, vol. 166, pp. 551-556, 2020.

[17] V. Sathya, S. M. Kala, S. Bhupeshraj, and B. R. Tamma, "Raptap: a socio-inspired approach to resource allocation and interference management in dense small cells," Wireless Networks, vol. 27, no. 1, pp. 441-464, 2021.

[18] S. K. Kasi, I. H. Naqvi, M. K. Kasi, and F. Yaseen, "Interference management in dense inband D2D network using spectral 
clustering \& dynamic resource allocation," Wireless Networks, vol. 25, no. 7, pp. 4431-4441, 2019.

[19] L. B. Kumar and P. Krishnan, "Multi-hop convergent fsouwoc system to establish a reliable communication link between the islands - sciencedirect," Optics Communications, vol. 474, 2020.

[20] D. T. Do, M. Nguyen, T. A. Hoang, and B. M. Lee, "Exploiting joint base station equipped multiple antenna and full-duplex $\mathrm{d} 2 \mathrm{~d}$ users in power domain division based multiple access networks," Sensors, vol. 19, no. 11, 2019.

[21] M. Aditya, H. Pancholi, P. Priyanka, and G. S. Kasbekar, "Beyond the vcg mechanism: truthful reverse auctions for relay selection with high data rates, high base station utility and low interference in $\mathrm{d} 2 \mathrm{~d}$ networks," Wireless Networks, vol. 26 , no. $6,2020$.

[22] I. Budhiraja, N. Kumar, and S. Tyagi, "Energy-delay tradeoff scheme for NOMA-based D2D groups with WPCNs," IEEE Systems Journal, vol. 9, no. 99, pp. 1-12, 2020.

[23] Y. Sun, M. Miao, Z. Wang, and Z. Liu, "Resource allocation based on hierarchical game for $\mathrm{d} 2 \mathrm{~d}$ underlaying communication cellular networks," Wireless Personal Communications, vol. 117, no. 2, pp. 281-291, 2021.

[24] M. Deypir and F. Gouya, "Multi-node d2d communications for wireless video delivery over cellular networks," $A E U$ International Journal of Electronics and Communications, vol. 110, Article ID 152863, 2019.

[25] M. Susanto, A. Abadi, Herlinawati, and A. Trisanto, "Uplink power control based on sinr for $\mathrm{d} 2 \mathrm{~d}$ enabled in cellular communication network," Journal of Physics: Conference Series, vol. 1376, Article ID 012019, 2019.

[26] R. A. Kumar and K. S. Prasad, "Performance analysis of gfdm modulation in heterogeneous network for $5 \mathrm{~g}$ nr," Wireless Personal Communications, vol. 439, 2020.

[27] H. V. Vu and T. Le-Ngoc, "Performance analysis of underlaid full-duplex d2d cellular networks," IEEE Access, vol. 7, pp. 176233-176247, 2019.

[28] A. Yx, A. Hz, and B. Pr, "Unified scheduling for predictable communication reliability in cellular networks with $\mathrm{d} 2 \mathrm{~d}$ links," Computer Communications, vol. 167, pp. 1-14, 2021.

[29] S. Kubota, H. Kawaki, B. Perbal, K. Kawata, T. Hattori, and T. Nishida, "Cellular communication network factor 3 in cartilage development and maintenance," Journal of Cell Communication and Signaling, pp. 1-11, 2021.

[30] S. Wang and M. Di Renzo, "On the mean interference-tosignal ratio in spatially correlated cellular networks," IEEE Wireless Communications Letters, vol. 9, no. 3, pp. 358-362, 2020.

[31] W. Santipach and K. Jiravanstit, "On selecting transmission mode for $\mathrm{d} 2 \mathrm{~d}$ transmitter in underlay cellular network with multi-antenna base station," Digital Communications and Networks, vol. 3, no. 3, 2021.

[32] W. Baohua, F. Shuyi, W. Cheng, and W. Qiang, "Optimization strategy of anti-interference performance based on bpso," Journal of Physics: Conference Series, vol. 1693, Article ID 012061, 2020.

[33] K. Kopsidas and M. Abogaleela, "Utilizing demand response to improve network reliability and ageing resilience," IEEE Transactions on Power Systems, vol. 34, no. 3, pp. 2216-2227, 2019.

[34] A. Iguider, O. Elissati, A. En-Nouaary, and M. Chami, "Shortest path method for hardware/software partitioning problems," International Journal of Information Systems and Social Change, vol. 12, no. 3, pp. 40-57, 2021.
[35] J. Gokulraj, J. Senthilkumar, Y. Suresh, and V. Mohanraj, "Data prediction approaches for efficient data transmission using optimized leibler distance matrix-based data aggregation in wireless sensor network," Journal of Ambient Intelligence and Humanized Computing, vol. 3, no. 26, pp. 1-8, 2021. 